\title{
Performance impact of IQ mismatch in direct-conversion MIMO OFDM transceivers
}

\author{
Tim C.W. Schenk ${ }^{* \ddagger}$, Erik R. Fledderus* and Peter F.M. Smulders* \\ * Eindhoven University of Technology, Radiocommunication Chair, PO Box 513, 5600 MB Eindhoven, The Netherlands \\ $\ddagger$ Philips Research, High Tech Campus 27, 5656 AE Eindhoven, The Netherlands, tim.schenk@philips.com.
}

\begin{abstract}
This contribution analytically studies the influence of transmitter (TX) as well as receiver (RX) IQ mismatch on the performance of multiple-antenna OFDM systems based on direct-conversion. Analytical expressions are derived for the probability of error for MIMO OFDM systems in both nonfaded and Rayleigh faded channels. The results can be used to derive matching specifications for the TX- and RX-branches. It is concluded that in fading channels RX IQ imbalance is on average more destructive than TX IQ imbalance. Additionally, it is concluded that the addition of extra $R X$ antennas is beneficial to reduce RX IQ imbalance dependence, but increases the TX IQ imbalance impact.
\end{abstract}

\section{INTRODUCTION}

The use of direct-conversion as basis for the implementation of multiple-input multiple-output (MIMO) OFDM, opens the door to low-cost implementations of this kind of systems. One of the disadvantages of direct-conversion transceivers is, however, that they can introduce considerable IQ imbalance [1-4].

While several previous contributions treat IQ imbalance in conventional single-input single-output (SISO) OFDM, they mainly rely on simulation results and signal-to-interference ratio (SIR) derivations, see e.g. [1], [2], to study the performance impact. Although these results reveal the performance impact to some extend, they do not provide analytical expressions for the final system performance measure, i.e., the error rate. Recently, the probability of bit error for SISO OFDM systems impaired by RX IQ imbalance was more accurately studied in [5]. The influence of IQ imbalance on the SIR performance of a space-time coded single-carrier system was treated, recently, in [4].

This paper extends previous work by considering the performance impact of both $T X$ and $R X I Q$ imbalance in a space division multiplexing ( $S D M)$ OFDM system. First the error in detection of the transmitted MIMO vector is derived in Section II. These results are in Section III applied to analytically derive novel expressions for the probability of symbol error for $M$-QAM signals. Section IV, subsequently, compares these analytical results with results from a numerical simulation study.

\section{ERROR IN SYMBOL DETECTION}

Consider a MIMO OFDM system with $N_{\mathrm{t}} \mathrm{TX}$ and $N_{\mathrm{r}} \mathrm{RX}$ branches and $N_{\mathrm{c}}$ subcarriers experiencing both TX and RX IQ imbalance. The $N_{\mathrm{r}} \times 1$ received frequency-domain symbol vector for the $k$ th carrier of the $m$ th symbol is given [6]:

$$
\begin{aligned}
\mathbf{x}_{m, k} & =\left(\mathbf{K}_{1} \mathbf{H}_{k} \mathbf{G}_{1}+\mathbf{K}_{2} \mathbf{H}_{-k}^{*} \mathbf{G}_{2}\right) \mathbf{s}_{m, k}+\mathbf{K}_{1} \mathbf{n}_{m, k} \\
& +\left(\mathbf{K}_{2} \mathbf{H}_{-k}^{*} \mathbf{G}_{1}^{*}+\mathbf{K}_{1} \mathbf{H}_{k} \mathbf{G}_{2}^{*}\right) \mathbf{s}_{m,-k}^{*}+\mathbf{K}_{2} \mathbf{n}_{m,-k}^{*}
\end{aligned}
$$

for the subset of $2 K$ modulated subcarriers, i.e., $k \in\{-K, \ldots,-1,1, \ldots, K\}$. Here $\mathbf{H}_{k}$ denotes the $N_{\mathrm{r}} \times N_{\mathrm{t}}$ quasi-static channel transfer matrix for the $k$ th subcarrier and * denotes complex conjugation. $\mathbf{s}_{m, k}$ and $\mathbf{n}_{m, k}$ denote the $N_{\mathrm{t}} \times 1$ TX symbol vector and $N_{\mathrm{r}} \times 1$ noise vector, respectively. It can be concluded from (1) that the received signal on the $k$ th carrier is a combination of the signal and noise vectors of carrier $k$ and its mirror carrier $-k$. In (1) the influence of TX IQ imbalance is contained in the $N_{\mathrm{t}} \times N_{\mathrm{t}}$ matrices $\mathbf{G}_{1}$ and $\mathbf{G}_{2}$ and that of RX IQ imbalance in the $N_{\mathrm{r}} \times N_{\mathrm{r}}$ matrices $\mathbf{K}_{1}$ and $\mathbf{K}_{2}$, which are defined as

$$
\begin{array}{ll}
\mathbf{G}_{1}=\left(\mathbf{I}+\boldsymbol{g}_{\mathrm{T}} e^{j \phi_{\mathrm{T}}}\right) / 2, & \mathbf{G}_{2}=\mathbf{I}-\mathbf{G}_{1}^{*}, \\
\mathbf{K}_{1}=\left(\mathbf{I}+\boldsymbol{g}_{\mathrm{R}} e^{-j \phi_{\mathrm{R}}}\right) / 2, & \mathbf{K}_{2}=\mathbf{I}-\mathbf{K}_{1}^{*},
\end{array}
$$

where $\mathbf{I}$ denotes the identity matrix. The imbalance matrices are defined as $\phi_{\mathrm{x}}=\operatorname{diag}\left\{\phi_{\mathrm{x}, 1}, \phi_{\mathrm{x}, 2}, \ldots, \phi_{\mathrm{x}, N_{\mathrm{x}}}\right\}$ and $\boldsymbol{g}_{\mathrm{x}}=\operatorname{diag}\left\{g_{\mathrm{x}, 1}, g_{\mathrm{x}, 2}, \ldots, g_{\mathrm{x}, N_{\mathrm{x}}}\right\}$, where $\mathrm{X} \in\{\mathrm{T}, \mathrm{R}\}$ and $N_{\mathrm{x}} \in\left\{N_{\mathrm{t}}, N_{\mathrm{r}}\right\}$. Here $g_{\mathrm{x}, n}$ and $\phi_{\mathrm{X}, n}$ are the amplitude and phase imbalance for the $n$th branch, respectively. When there are no imbalances, these parameters equal 1 and 0 , respectively.

When we assume perfect channel knowledge at the RX, the result of channel estimation will be given by the transfer from the TX baseband signals to the RX baseband signals and can be written as

$$
\tilde{\mathbf{H}}_{k}=\mathbf{K}_{1} \mathbf{H}_{k} \mathbf{G}_{1}+\mathbf{K}_{2} \mathbf{H}_{-k}^{*} \mathbf{G}_{2},
$$

where it is noted that the channel estimate now includes the influence of the TX and RX IQ imbalance. When this transfer is used for zero-forcing (ZF) based MIMO processing, the $N_{\mathrm{t}} \times 1$ estimated TX signal vector for the $k$ th subcarrier is found by

$$
\tilde{\mathbf{s}}_{m, k}=\tilde{\mathbf{H}}_{k}^{\dagger} \mathbf{x}_{m, k}=\mathbf{s}_{m, k}+\varepsilon_{k},
$$

where ${ }^{\dagger}$ denotes the pseudo-inverse and where it is easily verified that the error vector is given by

$$
\begin{aligned}
\varepsilon_{k}=\tilde{\mathbf{H}}_{k}^{\dagger}\left(\mathbf{K}_{2} \mathbf{H}_{-k}^{*} \mathbf{G}_{1}^{*}\right. & \left.+\mathbf{K}_{1} \mathbf{H}_{k} \mathbf{G}_{2}^{*}\right) \mathbf{s}_{m,-k}^{*} \\
& +\tilde{\mathbf{H}}_{k}^{\dagger}\left(\mathbf{K}_{1} \mathbf{n}_{m, k}+\mathbf{K}_{2} \mathbf{n}_{m,-k}^{*}\right) .
\end{aligned}
$$

We can conclude from (6) that the error term includes contributions of the signal term on the mirror carrier $-k$ and of the noise terms corresponding to subcarrier $k$ and $-k$.

When we now study the influence of TX and RX IQ imbalance, separately, we find that for a system only experiencing TX or RX IQ imbalance, the error term in (6) is given by

$$
\begin{aligned}
\boldsymbol{\varepsilon}_{\mathrm{T}, k} & =\mathbf{G}_{\mathrm{e}} \mathbf{s}_{m,-k}^{*}+\left(\mathbf{H}_{k} \mathbf{G}_{1}\right)^{\dagger} \mathbf{n}_{m, k}, \\
\boldsymbol{\varepsilon}_{\mathrm{R}, k} & =\mathbf{H}_{k}^{\dagger} \mathbf{K}_{\mathrm{e}} \mathbf{H}_{-k}^{*} \mathbf{s}_{m,-k}^{*}+\mathbf{H}_{k}^{\dagger}\left(\mathbf{K}_{\mathrm{e}} \mathbf{n}_{m,-k}^{*}+\mathbf{n}_{m, k}\right),
\end{aligned}
$$


respectively. Here $\mathbf{G}_{\mathrm{e}}$ and $\mathbf{K}_{\mathrm{e}}$ are diagonal matrices, defined as $\mathbf{G}_{\mathrm{e}}=\mathbf{G}_{1}^{\dagger} \mathbf{G}_{2}^{*}$ and $\mathbf{K}_{\mathrm{e}}=\mathbf{K}_{1}^{\dagger} \mathbf{K}_{2}$.

When comparing the error term due to TX IQ imbalance in (7) to that of RX IQ imbalance in (8), we conclude that the influence of noise in the error term is almost equal, although $\varepsilon_{\mathrm{R}, k}$ also exhibits a small noise contribution from the mirror. Considering the influence of the mirror leakage we can see that the term $\mathbf{G}_{\mathrm{e}} \mathbf{s}_{m,-k}^{*}$ in $\varepsilon_{\mathrm{T}, k}$ will be of finite size, but that $\mathbf{H}_{k}^{\dagger} \mathbf{K}_{\mathrm{e}} \mathbf{H}_{-k}^{*} \mathbf{s}_{m,-k}^{*}$ in $\boldsymbol{\varepsilon}_{\mathrm{R}, k}$ can become infinite large due to the channel matrices for carrier $k$ and $-k$. When through fading the elements of $\mathbf{H}_{-k}$ are large and the elements of $\mathbf{H}_{k}$ are small, the error term will become large. We can, thus, qualitatively conclude that in faded channels the influence of RX IQ imbalance will, on average, be greater than that of a comparable TX IQ imbalance.

\section{PROBABILITY OF ERRONEOUS DETECTION}

Although Section II provides a qualitative distinction between the influence of TX and RX IQ imbalance, a quantitative study is required to provide a more precise understanding of the impact on system performance. For that purpose we will, in this section, study the influence of the error vector $\varepsilon_{k}$ in (6) on the detection of the transmitted vector $\mathbf{s}_{m, k}$. We will calculate the probability of erroneous detection for transmit signals chosen from a rectangular $M$-QAM constellation. The influence is derived, separately, for TX and RX IQ imbalance in Sections III-A and III-B, respectively.

\section{A. TX IQ imbalance}

Let us first consider the case of TX IQ imbalance, where the error term $\varepsilon_{\mathrm{T}}$ is defined by (7). In our approach to calculate the probability of error, we will first consider the case for which the signal on the mirror carrier is given. We will treat this as a (known) translation of the wanted signal term, and later on average over all possible realisations of the mirror signal.

When we assume that the estimated signal on carrier $k$ in symbol $m$ is translated by the known $N_{\mathrm{t}} \times 1$ vector $\mathbf{d}_{m, k}=$ $\mathbf{G}_{\mathrm{e}} \mathbf{s}_{m,-k}^{*}$, the error in the estimated translated TX signal is found by combining $\mathbf{d}_{m, k}$ with (7), yielding

$$
\varepsilon_{\mathrm{T}, k}^{\prime}=\boldsymbol{\varepsilon}_{\mathrm{T}, k}-\mathbf{d}_{m, k}=\left(\mathbf{H}_{k} \mathbf{G}_{1}\right)^{\dagger} \mathbf{n}_{m, k}=\mathbf{H}_{k}^{\dagger} \mathbf{n}_{m, k}^{\prime} .
$$

Here $\mathbf{n}_{m, k}^{\prime} \sim \mathcal{C N}\left(\mathbf{0},\left(\sigma_{n}^{2} /\left|G_{1}\right|^{2}\right) \mathbf{I}\right)$ and we assumed the IQ imbalance to be equal on all TX branches, i.e., $\mathbf{G}_{1}=G_{1} \mathbf{I}$. Note that $\boldsymbol{\mu}_{\mathrm{T}}=\mathbb{E}\left[\varepsilon_{\mathrm{T}, k}^{\prime}\right]=\mathbf{0}$, since the mean of the elements of $\mathbf{n}_{m, k}$ equals zero and that the covariance matrix of the error in (9) is given by

$$
\boldsymbol{\Omega}_{\mathrm{T}}=\mathbb{E}\left[\boldsymbol{\varepsilon}_{\mathrm{T}, k}^{\prime}\left\{\boldsymbol{\varepsilon}_{\mathrm{T}, k}^{\prime}\right\}^{H} \mid \mathbf{G}_{1}, \mathbf{H}_{k}\right]=\frac{\sigma_{\mathrm{n}}^{2}}{\left|G_{1}\right|^{2}}\left(\mathbf{H}_{k}^{H} \mathbf{H}_{k}\right)^{-1} .
$$

The effective SNR for the $n_{\mathrm{t}}$ th branch and the $k$ th subcarrier can be found using (10) and is given by

$$
\wp_{n_{\mathrm{t}}, k}=\frac{\left|G_{1}\right|^{2} \sigma_{\mathrm{s}}^{2}}{\sigma_{\mathrm{n}}^{2}\left[\left(\mathbf{H}_{k}^{H} \mathbf{H}_{k}\right)^{-1}\right]_{n_{\mathrm{t}} n_{\mathrm{t}}}},
$$

where $[\mathbf{A}]_{m m}$ denotes the $m$ th diagonal element of matrix A and $\sigma_{\mathrm{s}}^{2}$ denotes the TX signal variance. When the channel matrix $\mathbf{H}_{k}$ has i.i.d. complex Gaussian entries, $\wp_{n_{\mathrm{t}}, k}$ is chisquare distributed with $2 R=2\left(N_{\mathrm{r}}-N_{\mathrm{t}}+1\right)$ degrees of freedom. The pdf of $\wp_{n_{\mathrm{t}}, k}$ is then given by

$$
p_{\wp_{n_{\mathrm{t}}, k}}(\rho)=\frac{\left(\rho / \wp_{0}\right)^{R-1}}{\wp_{0}(R-1) !} \exp \left(-\rho / \wp_{0}\right),
$$

where $\wp_{0}$ is the average SNR, given by $\wp_{0}=\left|G_{1}\right|^{2} \sigma_{\mathrm{s}}^{2} / \sigma_{\mathrm{n}}^{2}$.

In the remainder we will consider one carrier in one of the TX streams and, therefore, omit the subcarrier and branch index, i.e., we abbreviate $\wp_{n_{\mathrm{t}}, k}$ to $\wp$. Since the statistics are equal for all subcarriers and TX streams, the resulting probability of error can easily be generalised for all carriers later on. Due to independence, the probability of erroneous detection of $M$-QAM symbols can be found by the use of two $\sqrt{M}$-PAM signals, one for the real and one for the imaginary part. The probability of symbol error is then given by

$$
P_{\mathrm{e}, M \text {-QAM }, E_{\mathrm{S}}}^{d}=1-\left(1-P_{\mathrm{e}, \sqrt{M}-\mathrm{PAM}, E_{\mathrm{S}} / 2}^{d_{\mathrm{R}}}\right)\left(1-P_{\mathrm{e}, \sqrt{M}-\mathrm{PAM}, E_{\mathrm{s}} / 2}^{d_{\mathrm{I}}}\right),
$$

where $P_{\mathrm{e}, \sqrt{M}-\mathrm{PAM}, E_{\mathrm{S}} / 2}^{d_{\mathrm{R}}}$ and $P_{\mathrm{e}, \sqrt{M}-\mathrm{PAM}, E_{\mathrm{S}} / 2}^{d_{\mathrm{I}}}$ denote the probability of error for the translated $\sqrt{M}$-PAM modulation for the real and imaginary part of the $M$-QAM constellation, respectively. The power of these PAM signals is half of that of the QAM symbols. The real and imaginary part of the translation $d$ are given by $d_{\mathrm{R}}$ and $d_{\mathrm{I}}$, respectively.

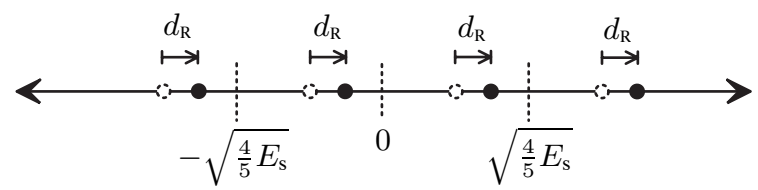

Fig. 1. Influence of $d_{\mathrm{R}}$-translation on the 4-PAM constellation.

The influence of a translation of $d_{\mathrm{R}}$ on the 4-PAM constellation is schematically depicted in Fig. 1. The original 4-PAM constellation points are depicted by dashed white dots, the translated symbols are depicted by black dots and the decision boundaries are depicted by dashed lines. It is clear from the figure that the distances to the decision boundaries are changed from the original $\sqrt{E_{\mathrm{s}} / 5}$. For half of the cases the distances now equals $\sqrt{E_{\mathrm{S}} / 5}-d_{\mathrm{R}}$ and the other half $\sqrt{E_{\mathrm{S}} / 5}+d_{\mathrm{R}}$, where the smallest one will impose the highest error probability.

When we now generalise the observations from Fig. 1, we find that for the real part the probability of error of an $N$-PAM systems is given by

$$
\begin{aligned}
P_{\mathrm{e}, N-\mathrm{PAM}, E_{\mathrm{s}}}^{d_{\mathrm{R}}} & =\frac{N-1}{2 N}\left(\mathbb{P}\left(\left|\varepsilon^{\prime}\right|>a_{1}-d_{\mathrm{R}}\right)+\mathbb{P}\left(\left|\varepsilon^{\prime}\right|>a_{1}+d_{\mathrm{R}}\right)\right) \\
& =\frac{N-1}{N}\left(Q\left(\frac{a_{1}-d_{\mathrm{R}}}{\sqrt{N_{0} / 2}}\right)+Q\left(\frac{a_{1}+d_{\mathrm{R}}}{\sqrt{N_{0} / 2}}\right)\right),(14)
\end{aligned}
$$

where $a_{1}=\sqrt{\frac{3 E_{\mathrm{s}}}{N^{2}-1}}, d_{\mathrm{R}}=\mathcal{R}\left\{G_{\mathrm{e}} s_{-k}^{*}\right\}, Q(x)$ denotes the $Q$-function and where $N_{0}$ denotes the noise power spectral density. A similar derivation for $P_{\mathrm{e}, N-\mathrm{PAM}, E_{\mathrm{S}}}^{d_{\mathrm{I}}}$ yields the same results as (14), where $d_{\mathrm{R}}$ is replaced with $d_{\mathrm{I}}=\mathcal{I}\left\{G_{\mathrm{e}} s_{-k}^{*}\right\}$. Substituting this into (13) yields

$$
\begin{aligned}
P_{\mathrm{e}, M-\mathrm{QAM}}^{d}= & 1-\left(1-c_{1}\left(\mathcal{U}\left(c_{2} \wp, \sqrt{2} d_{\mathrm{R}}\right)+\mathcal{U}\left(c_{2} \wp,-\sqrt{2} d_{\mathrm{R}}\right)\right)\right) \\
& \cdot\left(1-c_{1}\left(\mathcal{U}\left(c_{2} \wp, \sqrt{2} d_{\mathrm{I}}\right)+\mathcal{U}\left(c_{2} \wp,-\sqrt{2} d_{\mathrm{I}}\right)\right)\right),(15)
\end{aligned}
$$


where $\mathcal{U}(a, b)=Q\left(\sqrt{a}-\frac{b}{\sqrt{N_{0}}}\right), \quad c_{1}=1-1 / \sqrt{M}$, $c_{2}=3 /(M-1)$ and the SNR is defined as $\wp=E_{\mathrm{s}} / N_{0}$. For high SNR-values, (15) is well approximated by

$$
P_{\mathrm{e}, M \text {-QAM }}^{d} \approx c_{1}\left(\mathcal{U}\left(c_{2} \wp,-\sqrt{2} d_{\mathrm{R}}\right)+\mathcal{U}\left(c_{2} \wp,-\sqrt{2} d_{\mathrm{I}}\right)\right) .
$$

We note that (15) was calculated for a given $s_{-k}$, but that we aim at the averaged SER over all possible realisations of $s_{-k}$. Since the $d$ values originate from the $M$-QAM constellation, the probability of the occurrence of the different symbols is discrete uniform distributed. The average probability of error is thus given by

$$
P_{\mathrm{e}, M-\mathrm{QAM}}^{\mathrm{av}}=\frac{1}{M} \sum_{m=1}^{M} P_{\mathrm{e}, M-\mathrm{DAM}}^{d_{m}},
$$

where $d_{m}$ is the mirror interference term, which is a multiplication of the (given) $G_{\mathrm{e}}$ and the $m$ th symbol out of the $M$-QAM modulation alphabet.

We note that the probability of error was calculated above for a given SNR. To calculate the average SER for a faded channel the expressions for the SNR in (11) and (12) have to be applied. The SER for the $n_{\mathrm{t}}$ th branch and $k$ th subcarrier of the system is then found by

$$
P_{\mathrm{e}}=\int_{0}^{\infty} P_{\mathrm{e}, M-\text { QAM }}^{\mathrm{av}}(\rho) p_{\wp}(\rho) d \rho,
$$

where, again, the subcarrier and branch index were omitted for readability. The final expression for a MIMO OFDM system experiencing TX IQ imbalance and an i.i.d. Rayleigh faded channel is then found by substituting (12) and (17) into (18).

When we, as an example, work out the above for 4-QAM modulation, we find that the possible values of the translation are given by

$$
d_{m}=G_{\mathrm{e}} \frac{\sqrt{E_{\mathrm{s}}}( \pm 1 \pm j)}{\sqrt{2}}=\frac{1-g_{\mathrm{T}} e^{j \phi_{\mathrm{T}}}}{1+g_{\mathrm{T}} e^{j \phi_{\mathrm{T}}}} \frac{\sqrt{E_{\mathrm{s}}}( \pm 1 \pm j)}{\sqrt{2}},
$$

all with equal probability. It is then easily verified that due to symmetry $P_{\mathrm{e}, M-\mathrm{OAM}}^{d_{m}}$ is equal for all $m \in\{1, \ldots, 4\}$ and that the average probability of error in (17) is given by

$$
\begin{gathered}
P_{\mathrm{e}, 4-\text { OAM }}^{\mathrm{av}}=\frac{\mathcal{V}\left(\wp, g_{\mathrm{s}}\right)+\mathcal{V}\left(\wp,-g_{\mathrm{s}}\right)+\mathcal{V}\left(\wp, g_{\mathrm{D}}\right)+\mathcal{V}\left(\wp,-g_{\mathrm{D}}\right)}{2} \\
-\frac{\left[\mathcal{V}\left(\wp, g_{\mathrm{s}}\right)+\mathcal{V}\left(\wp,-g_{\mathrm{s}}\right)\right]\left[\mathcal{V}\left(\wp, g_{\mathrm{D}}\right)+\mathcal{V}\left(\wp,-g_{\mathrm{D}}\right)\right]}{4} .
\end{gathered}
$$

Here, we defined $\mathcal{V}(a, b)=Q(\sqrt{a}(1-b)), g_{\mathrm{s}}=g_{\mathrm{R}}+g_{\mathrm{l}}$, $g_{\mathrm{D}}=g_{\mathrm{R}}-g_{\mathrm{r}}$ and

$$
\begin{aligned}
g_{\mathrm{R}} & =\mathcal{R}\left\{G_{\mathrm{e}}\right\}=\left(1-g_{\mathrm{T}}^{2}\right) /\left(1+g_{\mathrm{T}}^{2}+2 g_{\mathrm{T}} \cos \left(\phi_{\mathrm{T}}\right)\right), \\
g_{\mathrm{I}} & =\mathcal{I}\left\{G_{\mathrm{e}}\right\}=-2 g_{\mathrm{T}} \sin \left(\phi_{\mathrm{T}}\right) /\left(1+g_{\mathrm{T}}^{2}+2 g_{\mathrm{T}} \cos \left(\phi_{\mathrm{T}}\right)\right) .
\end{aligned}
$$

The SER expression in (20) can be approximated for the high SNR region by

$$
\begin{aligned}
P_{\mathrm{e}, 4-\mathrm{QAM}}^{\mathrm{av}} \approx \frac{1}{2}[ & Q\left(\sqrt{\wp}\left(1-g_{\mathrm{s}}\right)\right)+Q\left(\sqrt{\wp}\left(1-g_{\mathrm{D}}\right)\right) \\
& \left.+Q\left(\sqrt{\wp}\left(1+g_{\mathrm{s}}\right)\right)+Q\left(\sqrt{\wp}\left(1+g_{\mathrm{D}}\right)\right)\right] .
\end{aligned}
$$

It can be concluded from (23) and Fig. 1 that the TX IQ imbalance will result in a shifting of the SER curves. Flooring will, however, only occur for IQ imbalance values where the translated constellation point are located outside the correct decision region. For even higher SNR values the last two terms in (23) can also be omitted.

The expressions in (20) and (23) provide the SER for a system experiencing TX IQ imbalance, but $n o$ fading channel. The SER for a system experiencing a fading channel can now be found by substituting (12) and either (23) or (20) into (18). A closed form SER expression can be found using a similar approach as we will use for RX IQ imbalance in Section III-B, yielding an expression based on hypergeometric functions.

\section{B. $R X I Q$ imbalance}

Let us, subsequently, consider the case of RX IQ imbalance for which the error vector $\varepsilon_{\mathrm{R}}$ is given by (8). The mean of this error term is given by $\boldsymbol{\mu}_{\mathrm{R}}=\mathbb{E}\left[\boldsymbol{\varepsilon}_{\mathrm{R}, k}\right]=\mathbf{0}$, since the elements of $\mathbf{s}_{m,-k}$ and $\mathbf{n}_{m, k}$ have zero mean. The covariance matrix for the error vector is given by

$$
\begin{aligned}
\boldsymbol{\Omega}_{\mathrm{R}} & =\mathbb{E}\left[\varepsilon_{\mathrm{R}, k} \varepsilon_{\mathrm{R}, k}^{H} \mid \mathbf{K}_{\mathrm{e}}, \mathbf{H}_{k}\right] \\
& =\left(\left|K_{\mathrm{e}}\right|^{2} N_{\mathrm{t}} \sigma_{\mathrm{s}}^{2}+\left(1+\left|K_{\mathrm{e}}\right|^{2}\right) \sigma_{\mathrm{n}}^{2}\right)\left(\mathbf{H}_{k}^{H} \mathbf{H}_{k}\right)^{-1},
\end{aligned}
$$

where we assumed $\mathbf{H}_{-k}$ to be independent of $\mathbf{H}_{k}$, which was shown in [5] to be a reasonable assumption for a practical system experiencing indoor multipath channels. Furthermore, we assumed in (24) that the IQ imbalance on all RX branches was equal, i.e., $\mathbf{K}_{\mathrm{e}}=K_{\mathrm{e}} \mathbf{I}$. The effective SNR for the $n_{\mathrm{t}}$ th branch and $k$ th carrier, is then found from (24) and given by

$$
\wp_{n_{\mathrm{t}}, k}=\frac{\sigma_{\mathrm{s}}^{2}\left\{\left[\left(\mathbf{H}_{k}^{H} \mathbf{H}_{k}\right)^{-1}\right]_{n_{\mathrm{t}} n_{\mathrm{t}}}\right\}^{-1}}{\left|K_{\mathrm{e}}\right|^{2} N_{\mathrm{t}} \sigma_{\mathrm{s}}^{2}+\left(1+\left|K_{\mathrm{e}}\right|^{2}\right) \sigma_{\mathrm{n}}^{2}} .
$$

Note again, that when the channel matrix $\mathbf{H}_{k}$ has i.i.d. complex Gaussian entries, $\wp_{n_{\mathrm{t}}, k}$ is chi-square distributed with $2 R=2\left(N_{\mathrm{r}}-N_{\mathrm{t}}+1\right)$ degrees of freedom and that its pdf is given by (12). The average (effective) SNR is here given by

$$
\wp_{0}=\sigma_{\mathrm{s}}^{2} /\left\{\left|K_{\mathrm{e}}\right|^{2} N_{\mathrm{t}} \sigma_{\mathrm{s}}^{2}+\left(1+\left|K_{\mathrm{e}}\right|^{2}\right) \sigma_{\mathrm{n}}^{2}\right\} .
$$

The SER for the $n_{\mathrm{t}}$ th branch and $k$ th subcarrier of an uncoded system is then found using (18), where $P_{\mathrm{e}, M \text {-OAM }}^{\mathrm{av}}=P_{\mathrm{e}, M \text {-OAM }}$ is the approximation of the SER for an $M$-QAM constellation and it is given by $P_{\mathrm{e}, M \text {-OAM }}(\wp)=c_{3} Q\left(\sqrt{c_{2} \wp}\right)$. In this expression $c_{3}=4(1-1 / \sqrt{M})$. The average SER is now found by averaging $P_{\mathrm{e}}$ over the different subcarriers and branches.

To derive a closed form expression for the SER, we use an alternative representation for the Gaussian $Q$-function [7, p.71]. By substituting this expression and (12) into (18), working out one integral and by change of integration variable, we find that the probability of symbol error is given by

$$
P_{\mathrm{e}}=\frac{c_{3}\left(1-\sqrt{\wp_{0}} c_{4}\right)}{2}{ }_{2} F_{1}\left(\frac{1}{2}, R+\frac{1}{2} ; \frac{3}{2} ; \frac{-c_{2} \wp_{0}}{2}\right),
$$

where $c_{4}=\sqrt{\frac{2 c_{2}}{\pi}} \frac{\left(R-\frac{1}{2}\right) !}{(R-1) !}$ and ${ }_{2} F_{1}$ denotes the hypergeometric function. 


\section{NUMERICAL RESULtS}

The analytical results derived in Section III are here compared with results from Monte Carlo simulations. All simulations are carried out for an uncoded IEEE 802.11a-based MIMO system. It is assumed that the MIMO transfer is perfectly estimated, yielding the estimate expressed in (4). All figures presented in this section depict the analytical results by lines and the results from simulations by markers.

First the influence of TX IQ imbalance in a non-fading channel is studied. To that end a SISO system applying 4-QAM and 64-QAM modulation is considered. The SER results are reported in Fig. 2 together with the analytical results found for 4-QAM using (20) (solid) and (23) (dashed), and for 64-QAM using (17). The results are depicted as function of the average SNR per RX antenna, which is given by $N_{\mathrm{t}} \sigma_{\mathrm{s}}^{2} / \sigma_{\mathrm{n}}^{2}$.
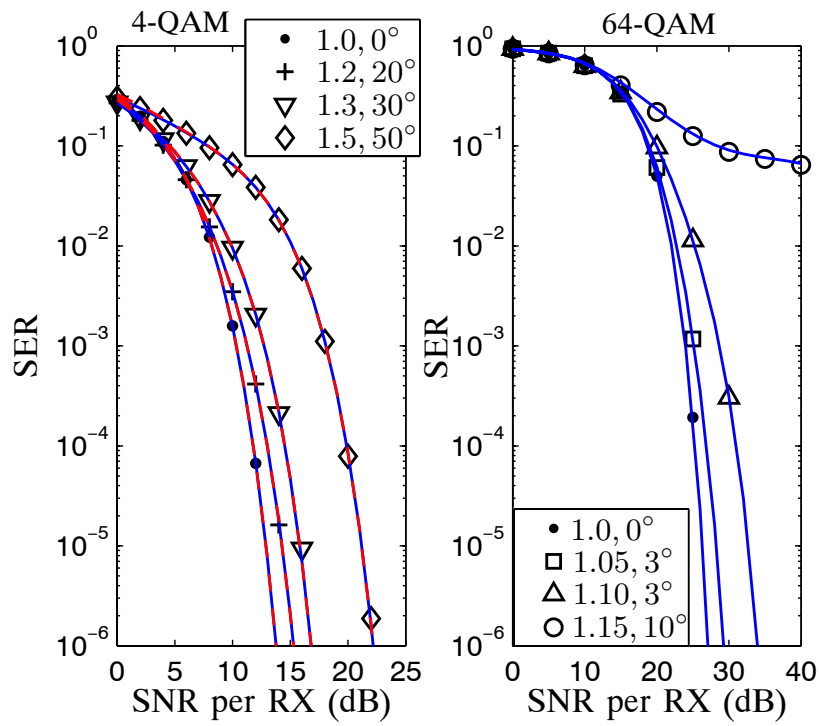

Fig. 2. SER performance of a system experiencing TX IQ imbalance and an AWGN channel. Results are depicted for an $1 \times 1$ 4-QAM (left) and 64-QAM (right) system. The legend reports the IQ imbalance parameters $\left\{g_{\mathrm{T}}, \phi_{\mathrm{T}}\right\}$.

It can be concluded from Fig. 2 that the analytical results accurately predict the SER results found from simulations, proving the applicability of the derived expressions. It can furthermore be concluded that considerable degradation only occurs for very high values of IQ imbalance. Additionally, although degradation in SER performance occurs, flooring only seems to occur for 64-QAM and an IQ imbalance of $g_{\mathrm{T}}=1.15$ and $\phi_{\mathrm{T}}=10^{\circ}$, i.e., for values of the translation $d$ where the means of some of the constellation points are shifted outside their nominal decision regions.

The SER results for different 4-QAM MIMO systems experiencing IQ imbalance and a channel with independent Rayleigh fading per subcarrier are depicted in Fig. 3. The figure compares the analytical results of (18) and (27) with simulation results for TX and RX IQ imbalance, respectively. It is observed from this figure that also for the fading channel the analytical expressions accurately predict the error performance. It is concluded that the influence of the (high) TX IQ imbalance on the performance of the symmetric MIMO systems is small, since in none of the considered cases flooring in the SER performance occurs. It is furthermore concluded that the degradation due to the, much lower, RX IQ imbalance is considerably higher than that of TX IQ imbalance, as was already qualitatively concluded in Section II. It is noted, additionally, that these small values of RX IQ imbalance already cause flooring in the SER performance, in contrast to TX IQ imbalance, which can be explained by the nature of the error term. The level of flooring depends on the MIMO configuration. We note that going from a $2 \times 2$ to a $2 \times 4$ system decreases the RX IQ imbalance dependence, but increases the TX IQ imbalance impact.

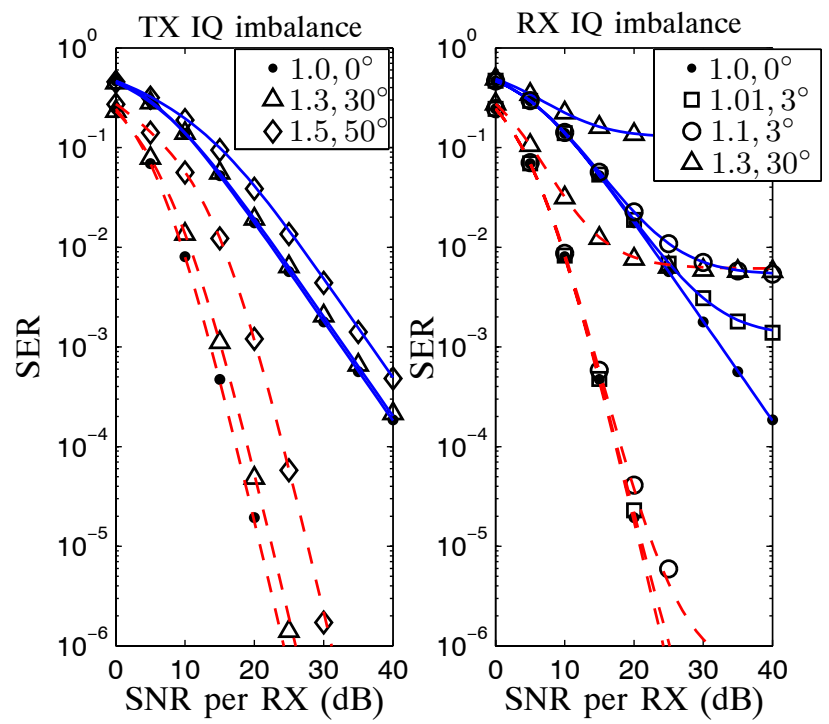

Fig. 3. SER performance of a system experiencing TX (left) or RX (right) IQ imbalance and a Rayleigh faded channel. Results are depicted for a $2 \times 2$ (solid lines) and a $2 \times 4$ (dashed lines) 4-QAM system. The legend reports the IQ imbalance parameters $\left\{g_{\mathrm{T}}, \phi_{\mathrm{T}}\right\}$ and $\left\{g_{\mathrm{R}}, \phi_{\mathrm{R}}\right\}$, respectively.

\section{CONCLUSIONS}

The impact of IQ imbalance on the performance of a multiple-antenna OFDM system was studied in this paper. Both the error in detection and the SER performance were derived analytically. RX IQ imbalance was found to be, on average, the dominant error source.

\section{REFERENCES}

[1] C.-L. Liu, "Impacts of I/Q imbalance on QPSK-OFDM-QAM detection," IEEE Trans. Consum. Elect., vol. 44, no. 3, pp. 984-989, Aug. 1998.

[2] M. Buchholz, A. Schuchert, and R. Hasholzner, "Effects of tuner IQ imbalance on multicarrier-modulation systems," in Proc. the 2000 3th IEEE ICCDCS, March 2000, pp. T65/1 - T65/6.

[3] M. Valkama, M. Renfors, and V. Koivunen, "Advanced methods for I/Q imbalance compensation in communication receivers," IEEE Trans. on Signal Proc., vol. 49, pp. 2335-2344, Oct. 2001.

[4] M. Valkama, Y. Zou, and M. Renfors, "On I/Q imbalance effects in MIMO space-time coded transmission systems," in Proc. IEEE RWS 2006, Jan. 2006, pp. 223-226.

[5] M. Windisch and F. Fettweis, "Performance degradation due to $\mathrm{I} / \mathrm{Q}$ imbalance in multi-carrier direct conversion receivers: A theoretical analysis," in Proc. IEEE ICC 2006, June 2006.

[6] T. C. W. Schenk, P. F. M. Smulders, and E. R. Fledderus, "Estimation and compensation of TX and RX IQ imbalance in OFDM based MIMO systems," in Proc. IEEE RWS 2006, Jan. 2006, pp. 215-218.

[7] M. K. Simon and M. S. Alouni, Digital Communication over Fading Channels, A Unified Approach to Performance Analysis, Wiley, NY, 2000. 